la revue La revue pour l'histoire du CNRS

POUR LHISTORE DU CNRS $\quad 24 \mid 2009$

Soixante-dixième anniversaire du CNRS

\title{
Risques : la fabrique d'une histoire
}

Anne-Marie Granet-Abisset

\section{OpenEdition}

Journals

Édition électronique

URL : https://journals.openedition.org/histoire-cnrs/9126

DOI : 10.4000/histoire-cnrs.9126

ISSN : 1955-2408

Éditeur

CNRS Éditions

Édition imprimée

Date de publication : 5 octobre 2009

ISSN : 1298-9800

Référence électronique

Anne-Marie Granet-Abisset, "Risques : la fabrique d'une histoire », La revue pour l'histoire du CNRS [En ligne], 24 | 2009, mis en ligne le 05 octobre 2009, consulté le 20 mai 2021. URL : http://

journals.openedition.org/histoire-cnrs/9126; DOI : https://doi.org/10.4000/histoire-cnrs.9126

Ce document a été généré automatiquement le 20 mai 2021.

Comité pour l'histoire du CNRS 


\title{
Risques : la fabrique d'une histoire
}

\author{
Anne-Marie Granet-Abisset
}

1 Exception faite d'Emmanuel Le Roy Ladurie, que son Histoire du climat depuis l'an mil consacrait grand spécialiste, ou de certains médiévistes et modernistes et de leurs travaux novateurs sur les calamités, les malheurs du temps ou plus largement l'environnement, le thème n'était pas réellement fédérateur. Pire, il n'inspirait que peu de chercheurs. D'une manière générale, ce champ de recherches était concédé à d'autres sciences humaines (géographie, sociologie, sciences politiques) sans doute mieux armées méthodologiquement et épistémologiquement pour s'en saisir. «Le pôle grenoblois sur les risques naturels, véritable espace de synergie fédérant les chercheurs autant que les laboratoires, a sans doute très largement contribué à l'inscription de ce sujet dans mes recherches comme à la mise en place d'une équipe d'historiens sur ce thème ", explique Anne-Marie Granet-Abisset.

2 «En tant que spécialiste de la mémoire en histoire et du travail sur et avec les témoins, mes premiers travaux de recherche sur les migrations des populations alpines aux XIX ${ }^{\mathrm{e}}$ et $\mathrm{XX}^{\mathrm{e}}$ siècles avaient montré que le rapport au territoire était bien différent de celui habituellement stigmatisé dans les écrits sur le sujet », ajoute-t-elle. " Au contraire, des enquêtes précises et une approche de type microstoria avaient fait ressortir leurs connaissances précises des risques inhérents à ces territoires dangereux. » Ce savoir maîtrisé, associant la pratique du terrain et la transmission des événements dramatiques, générait des comportements en matière d'habitat, de circulation et d'entretien du foncier très éloignés de la fatalité et de la passivité, descriptifs classiques pour ces montagnards, rangés jusqu'il y a peu dans le camp du retard et de l'archaïsme.

3 «Étant convaincue de l'apport évident de l'histoire et des historiens sur ces thématiques, j'ai accepté avec enthousiasme la sollicitation de chercheurs du Cemagref de Grenoble pour participer à un programme de recherche interdisciplinaire sur les avalanches. La commande portait sur la collecte de témoignages sur les savoirs en matière d'avalanches (tracé, événements, localisation, gestion) qui devaient nourrir une base de données historiques sur le sujet », souligne A.-M. Granet-Abisset. Ce projet, intéressant à plusieurs titres, a ainsi permis de tester les questions mémorielles sur des sujets inédits et peu attendus en histoire et d'établir un partenariat avec des 
chercheurs de sciences exactes, voire des chercheurs insérés dans des programmes de recherche-action. Aux problématiques classiques de la mémoire (les savoirs, les modes de transmission, les formes du récit, les oublis et les silences intéressés ou inconscients), ou moins habituelles (la peur, les marqueurs territoriaux), s'ajoutaient les aspects méthodologiques inhérents aux usages des bases de données et à leurs limites. La discipline, jusque-là trop souvent confinée au rôle de vecteur d'histoire des sciences et des techniques, d'auxiliaire des autres disciplines, de pourvoyeuse de documents, de déchiffreuse de sources anciennes, pouvait enfin faire reconnaître sa légitimité dans des programmes interdisciplinaires. C'est le cas en particulier de l'histoire de la période contemporaine où la fonction est occupée par les sociologues ou les géographes.

4 «J'ai eu à formuler et expliciter les bases de notre discipline - méthodes, démarches, problématiques, objets d'étude - pour l'inscrire comme une discipline scientifique à part entière et combattre la vision pour le moins réductrice de certains de mes collègues sceptiques ", analyse A.-M. Granet- Abisset. «Le travail avec les sciences dures, peu habituel pour l'historien, oblige à ces positionnements. Quand chacun fait montre d'ouverture, de compréhension et d'égalité, l'interdisciplinarité est féconde et stimulante pour les chercheurs. » L'emprunt aux autres sciences et la connaissance des autres démarches sont productifs, pas la dilution dans une discipline mixte qui n'aboutit qu'à un affaiblissement de toutes les disciplines. Enfin, cette thématique des risques a révélé des résultats forts instructifs.

5 Plus encore, cette histoire sociale des risques a permis de « re-questionner » les travaux précédents, nourri d'autres entrées sur les objets de recherches antérieurs et suscité de nouveaux sujets, notamment à propos des sociétés de montagne, un beau laboratoire d'études. Les risques dits naturels n'intéressent l'historien que lorsqu'ils arrivent dans des territoires habités (que ces risques et les événements catastrophiques associés soient d'origine physique ou anthropique). Or, ils sont une clef pertinente de compréhension des sociétés et des comportements humains, des représentations qui les génèrent et des politiques de gestion que les différents acteurs choisissent, acceptent ou refusent d'appliquer.

6 À l'inverse, on ne peut décoder et analyser ces comportements en matière de risques que lorsqu'on les réinsère dans une approche globale des sociétés dont la connaissance est fondamentale pour poser les hypothèses les plus pertinentes et fonder des analyses aussi justes que possible. "C'est dans cette dialectique que se nourrissent des questionnements et des hypothèses riches qui peuvent le mieux contribuer à apporter des résultats aux historiens comme à d'autres disciplines", conclut A.-M. GranetAbisset.

\section{RÉSUMÉS}

À la fin des années 1990, qu'un historien s'empare du thème des risques naturels pour en faire son objet de recherche ne semblait pas aller de soi. Les risques : un sujet pour les historiens? 
Anne-Marie Granet- Abisset s'interroge sur les contingences de la recherche qui, si elles relèvent souvent de phénomènes scientifiques, doivent beaucoup au contexte, aux réseaux de relations et aux aptitudes à emprunter des chemins de traverse.

\section{AUTEUR}

ANNE-MARIE GRANET-ABISSET

Anne-Marie Granet-Abisset est professeur d'histoire contemporaine à l'université de Grenoble et membre du Laboratoire de recherche historique Rhône-Alpes (Larhra). 